\title{
Conus medullaris trauma: is there a greater risk in parturients?
}

Julie Verkooijen, Hilde Coppejans, Els Mertens, Vera Saldien, Marcel Vercauteren

Antwerp University Hospital, Department of Anaesthesia, Edegem, Belgium

\begin{abstract}
Background. There is some evidence that anaesthetists often perform neuraxial blocks at a higher lumbar interspace than intended. It may be questioned whether parturients are at greater risk for neurological damage when the dura is perforated at a more cephalad interspace than L2-L3.

Methods. Thirty-six patients scheduled for elective Caesarean delivery under CSE anaesthesia were selected for study. Using a B-D Durasafe Adjustable needle combination, the skin-to-epidural distance and the width of the epidural space were measured and matched with 36 female patients undergoing the same anaesthetic technique for orthopaedic procedures.

Results. Pregnant patients had a higher bodyweight ( $77 \mathrm{vs.} 67 \mathrm{~kg}, \mathrm{p}=0.007)$ than those scheduled for orthopaedic surgery. The skin-to-epidural distance was similar in both groups (5.3 vs. $5.1 \mathrm{~cm}, \mathrm{p}=0.3)$. The width of the epidural space was $1.1 \mathrm{~mm}$ larger in parturients $(8.2 \mathrm{vs} .7 .1 \mathrm{~mm}, \mathrm{p}=0.04)$. More patients in this group had tip-to-tip distances exceeding $10 \mathrm{~mm}$ (25 vs. $12 \%)$.

Conclusion. The greater epidural space or tip-to-tip distance between the epidural and spinal needle points in term parturients results in a lower margin of safety with respect to the distance from the dura to spinal cord or conus medullaris. Puncturing the correct interspace is, therefore, of crucial importance in pregnant patients.
\end{abstract}

Keywords: anaesthesia, spinal, epidural, complications, neurological, conus medullaris

\section{Introduction}

Reynolds reported seven patients suffering persistent neurological damage caused by a lesion of the conus medullaris, possibly due to spinal needle trauma [1]. In four of the seven cases, dural puncture was performed during a Combined Spinal-Epidural (CSE) technique, with six of the patients being parturients. Fifteen years ago, this report warned of the need for care with dural puncture, but in reality our daily practice

Address for correspondence: Marcel Vercauteren, MD, $\mathrm{PhD}$ Antwerp University Hospital, Department of Anaesthesia Wilrijkstraat 10 , 2650 Edegem, Belgium E-mail: marcel.vercauteren@uza.be has remained unchanged. The increasing number of malpractice reports of conus medullaris damage resulting in litigation raises pertinent questions with regard to the dangers of puncturing the L2-L3 interspace, the value of Tuffier's line and the possible benefit of ultrasound scanning.

Although it is known that the spinal cord and Tuffier's line may extend more caudally in females as compared to males [2], the most important reason giving rise to conus medullaris trauma is too high needle insertion by the anaesthetist. As a consequence, since palpation of the vertebral spinous processes can lead to erroneous clinical assessment of the intervertebral spaces, spinal cord trauma may occur during puncture at the perceived L1-L2 level. Indeed, when a number of anaesthetists were asked to identify the L2-L3 intervertebral space by locating Tuffier's line, considerable variability was observed, with many anaesthetists tending to select an interspace one or more dermatomes more cephalad [3]. 
In this study we aimed to evaluate whether, as compared to non-pregnant females, the protrusion distance of the spinal needle tip beyond the Tuohy needle orifice might be greater in parturients and as a consequence enhance the risk to damage the conus in case the anaesthetist would have selected a too cephalad interspace.

\section{Methods}

During a six month period we studied women who were to undergo elective Caesarean section at term and matched female patients undergoing orthopaedic in-hospital surgery under CSE anaesthesia. Recruitment of a matched orthopaedic patient occurred following each Caesarean patient on a one-to-one basis, and all anaesthetic procedures were performed by the same anaesthetists. Patients were excluded from study on account of extreme height $(<150 \mathrm{~cm}$ and $>180 \mathrm{~cm}$ ) and/or weight $(>100 \mathrm{~kg}$ ), and a history of previous back surgery. All patients gave informed consent and the protocol was approval by the hospital ethics committee.

During the study period ultrasound had not yet become available. CSE was performed with patients in the right lateral decubitus position at an intended interspace of L3-L4 or L4-L5. The Durasafe Adjustable combination needle set (Becton Dickenson, Barcelona, Spain) was used.

With the patient in the lateral decubitus position a skin wheel was raised at either the L3-L4 lumbar interspace. In the midline approach the epidural space was identified with the Tuohy needle using loss-ofresistance to air. At the moment of loss of resistance, the distance travelled by the Tuohy needle was measured to the nearest $1 \mathrm{~mm}$ based on the calibration upon the epidural needle shaft. Although not entirely correct it was named the skin-to-epidural distance (SED).

The $27 \mathrm{G}$ Whitacre spinal needle was introduced using a needle-through-needle technique. With this device, the spinal needle was in the zero position when its tip was located in the orifice of the Tuohy needle. By means of a push-button grip adapter the spinal needle was advanced to leave the Tuohy needle orifice. A click could then be felt as the advancing spinal needle penetrated the dura. The button on the spinal needle was then deployed, thus fixing it in position. The stylet was then removed to confirm backflow of CSF. If this did not occur, the stylet was replaced and the spinal needle further advanced until the dura was penetrated. After appearance of CSF, the local anaesthetic solution was injected. The stylet was then reintroduced and the spinal needle removed while taking care not to touch the button or withdraw the needle into its zero position.
Following placement of the epidural catheter and removal of the Tuohy needle, the distance between the two needle tips (the tip-to-tip distance, equivalent to the width of the epidural space) could then be measured to the nearest $0.1 \mathrm{~mm}$ using a compass calliper.

Based on mean values and standard deviations obtained in previous studies 34 patients were required in each group to obtain a TTD difference of at least $1.0 \mathrm{~mm}$ and a power of $80 \%$. Statistical analysis was performed with the two-tailed Student-t test (mean, SD) and Mann-Whitney-U test (non-parametric data). A $p$ value $<0.05$ was considered to be significant.

\section{Results}

In case of some patients being discarded from the study, 36 subjects in each group were selected for participation in the study. All procedures were performed by Board Certified anaesthesiologists with at least 5 years of experience with the CSE technique and the particular device used.

All blocks were successful and uneventful. No paraesthesias were observed and a click sensation on dural puncture was experienced in $>90 \%$ of the patients. Supplementary dosing of local anaesthetic in the epidural catheter was never required. None of the patients experienced a paraesthesia. Free flow of cerebrospinal fluid was obtained in all subjects.

The ages and height of the patients did not differ between the two groups (Table 1). Not unexpectedly, the pregnant patients had a significantly higher body weight than their non-pregnant counterparts, but despite a higher BMI, the somewhat longer skin-to-epidural distance was not significantly different between the groups. However, the tip-to-tip distance (epidural space width) appeared to be significantly greater in the pregnant group. More patients in the pregnant group had tip-to-tip distances exceeding $10 \mathrm{~mm}$, but this difference did not reach statistical significance $(25 \%$ vs. $12 \%, \mathrm{p}=0.07$ ).

Table 1. Demographic and anatomical data*

\begin{tabular}{lccc}
\hline & $\begin{array}{c}\text { Pregnant } \\
\text { Group }\end{array}$ & $\begin{array}{c}\text { Orthopaedic } \\
\text { Group }\end{array}$ & P Value \\
\hline Age $(\mathrm{yrs})$ & $30(5)$ & $32(5)$ & 0.09 \\
Height $(\mathrm{cm})$ & $164(6)$ & $166(6)$ & 0.40 \\
Weight $(\mathrm{kg})$ & $77(17)$ & $67(15)$ & 0.007 \\
SED $(\mathrm{cm})$ & $5.3(1)$ & $5.1(1)$ & 0.30 \\
TTD $(\mathrm{mm})$ & $8.2(2.1)$ & $7.1(2.2)$ & 0.04 \\
\hline
\end{tabular}

* Data are expressed as mean (SD). SED: Skin to Epidural space distance, TTD: tip-to-tip distance 


\section{Discussion}

The present study found that the tip-to-tip distance (TTD) in pregnant patients was significantly greater than in non- pregnant patients. Only one similar study has also measured possible differences between a pregnant and non-pregnant population but the difference of $0.8 \mathrm{~mm}$ was not found to be significant [4]. However Tanaka et al used both classical and lockable Combined Spinal-Epidural (CSE) devices which may have affected the accuracy of their measurements.

Bevacqua et al. tried to use MRI to determine the distance between the ligamentum flavum and the dura [5]. In 150 subjects we previously used the same Durasafe Adjustable combination needle set to measure the tip-to-tip distance and to compare these measurements with those obtained by imaging techniques [6]. Despite a mean value of $7-8 \mathrm{~mm}, 13 \%$ of patients were found to have a distance exceeding $10 \mathrm{~mm}$, while a further $20 \%$ had values lower than $5 \mathrm{~mm}$. This may be explained by anatomical differences and by the angulation at which the epidural space is traversed. The skin to epidural (SED) distance was larger with higher BMI and body weight values while the correlation between the tip-to-tip distance and body weight / BMI was much weaker (because, contrary to what might be anticipated, obese patients do not have more epidural fat) [6]. In the present study we could not demonstrate an effect of the BMI or body weight upon the less accurate SED measurement.

Compared to non-pregnant subjects, identification of the appropriate lumbar interspace in pregnancy may be more difficult due to increased fat accumulation around the hips affecting the position of Tuffier's line and the increase in abdominal volume decreasing the mother's ability to curve her back.

It may be questioned whether there is a higher risk of mistaking the lumbar interspaces in pregnant patients, and if so, whether this leads to greater consequences than in non parturients.

Many studies have looked at the accuracy of clinical examination in determining the level of the intervertebral spaces. Broadbent et al. demonstrated that anaesthetists were very poor at determining intervertebral spaces, with only $29 \%$ identifying the level correctly [3]. Schlotterbeck et al. tried to assess the reliability of the usual anatomical landmarks in identifying the intervertebral puncture level for neuraxial anaesthesia in labouring patients [7]. They observed that the intended intervertebral level corresponded to the actual level, as verified by ultrasound, in only $36.4 \%$ of patients. The puncture level was often more cephalad than clinically estimated with 6 punctures out of 99 performed at the level L1/L2.

Based on our present results, the pregnant patient has a deeper posterior epidural space, which explains why pregnancy may increase the risk of spinal cord trauma. Since the surrounding bony structures are relatively rigid, the larger epidural fat mass and engorgement of epidural veins can more easily compress the softer intra-vertebral anatomy. Therefore, the deeper epidural space can only be accommodated by a reduction in CSF volume. The margin of safety for avoiding needle penetration of the conus is thus reduced by the narrower space between the dura and spinal cord. It may therefore be questioned whether spinal anaesthesia at the L2-L3 interspace should be avoided in order to prevent a needle puncturing the dura at the L1-L2 interspace or even higher.

Commonly, anaesthetists tend to select the interspaces L2-L3 down to L4-L5 for performing spinal or epidural anaesthesia. Studies in non-pregnant patients have compared the different clinical characteristics between spinal anaesthesia performed at L2-L3 and L4-L5. Chin et al. showed in his study that injection at L2-L3 produced a significantly higher mean maximum spread of analgesia (T6) when compared to injection at L4-L5 (T10) [8]. Similar results were obtained in the study by Tuominen et al. Injection at L2-L3 also produced a significantly higher spread of analgesia (T7) than injection at L4-L5 (T11) [9].

In a similar study by Lowson et al. using bupivacaine, the onset of anaesthesia at T10 and T6 was faster following injection at the higher interspace, although the final sensory level was not different [10]. The higher extent of the upper sensory block may explain why anaesthetists often prefer to perform spinal anaesthesia at a higher interspace, which is often anatomically easier than at lower levels. Although these studies did not verify if the selected interspace corresponded with the real lumbar level, no sequelae were reported.

In 2000, Reynolds stated that "The L2-L3 level should not be an option and several textbooks need to be rewritten to allow for human variability" [11]. According to a study by Srinivasan et al. it is best to avoid the L2-L3 interspace or higher, and to go one interspace down from the Tuffier's line when this crosses either an interspace or spinous process [12].

Although there are some arguments for avoiding the L2-L3 interspace based on anatomical landmarks, is it also the case that this interspace should not be chosen when the level can be verified by imaging techniques? During recent years the use of ultrasound has been recommended, but we are still far from seeing its routine or even compulsory use for normal, unoperated and non-obese backs. According to Bogod, it requires moderately little training to be able to determine spinal level ultrasonically using a paramedian sagittal view to identify the sacrum and its cranial transition into separate lumbar vertebrae at the L5 level. It is then a simple matter of counting upwards to identify 
the different interspaces [13]. But this technique is not failsafe - the patient may have a sacralisation of L5 or a lumbalisation of S1.

Although not specifically studied, our experience with CSE and measurement of the epidural space depth may also suggest why CSE anaesthesia may carry a greater risk for neurological damage than a single dose spinal. As a single dose spinal injection is performed without a previous injection of air or saline in the epidural space, this may leave sufficient distance from the dura to the spinal cord. The method of identifying the epidural space may also affect the tip-to-tip distance measured, as the actual epidural space may be enlarged by the injection of air or saline. The tip-to-tip distance was found to be $2 \mathrm{~mm}$ longer when using a loss of resistance to air technique as compared to the hanging-drop technique [14]. Unfortunately, no study has compared air (as used for CSE in many departments) with saline. In addition, as the equipment used for CSE procedures requires some experience, the click feeling may be felt less frequently than with a single dose spinal. We demonstrated that starting to use new equipment such as a lockable needle requires a learning curve to obtain a click-feeling in $>90 \%$ of the patients [6]. Adecreased feeling of a dural click may cause the anaesthetist to introduce the spinal needle more deeply than with a single dose spinal technique.

Finally, as anaesthetists use pencil-point needles for dural puncture, the presence of a blind tip requires the spinal needle to be inserted further into the CSF when compared with Quincke needles. This may also explain why even with the spinal needle tip in the conus, free flow of CSF may still occur.

In conclusion, the wider epidural space in pregnant patients, along with previously injected air or saline, may explain why pregnancy enhances the risk of perforating or damaging the conus medullaris during CSE. Furthermore, when providing CSE, there is a need for certainty that the appropriate level has indeed been selected, especially if we choose the L2/3 interspace.

\section{Conflict of interest}

Nothing to declare

\section{Acknowledgements}

The authors would like to thank Stuart Morrison MD for language correction and critical consideration of the manuscript.

\section{References}

1. Reynolds F. Damage to the conus medullaris following spinal anaesthesia. Anaesthesia 2001; 56: 238-247. DOI: 10.1046/ j.1365-2044.2001.01422-2.x

2. Kim JT, Bahk JH, Sung J. Influence of age and sex on the position of the conus medullaris and Tuffier's line in adults. Anesthesiology 2003; 99: 1359-1363

3. Broadbent CR, Maxwell WB, Ferrie R, Wilson DJ, Gawne-Cain M, Russell R. Ability of anaesthetists to identify a marked lumbar interspace. Anaesthesia 2000; 55: 1122-1126. DOI: 10.1046/ j.1365-2044.2000.01547-4.x

4. Tanaka N, Ohkubo S, Takasaki M. Evaluation of a lockable combined spinal-epidural device for use with needle-throughneedle technique. Masui 2004; 53: 173-177

5. Bevacqua BK, Haas T, Brand F. A clinical measure of the posterior epidural space depth. Reg Anesth 1996; 21: 456-460

6. Hoffmann VL, Vercauteren MP, Buczkowski PW, Vanspringel GL. A new combined spinal-epidural apparatus: measurement of the distance to the epidural and subarachnoid spaces. Anaesthesia 1997; 52: 350-355. DOI: 10.1111/j.1365-2044.1997.93az0093. $\mathrm{x}$

7. Schlotterbeck H. Schaeffer R., Dow WA, Touret Y, Bailey S, Diemunsch P. Ultrasonographic control of the puncture level for lumbar neuraxial block in obstetric anaesthesia. Br J Anaesth 2008; 100: 230-234. DOI: 10.1093/bja/aem371

8. Chin KW, Chin NM, Chin MK. Spread of spinal anaesthesia with $0.5 \%$ bupivacaine: Influence of the vertebral interspace and speed of injection. Med J Malaysia 1994; 49: 142-148

9. Tuominen M, Taivainen T, Rosenberg PH. Spread of spinal anaesthesia with plain $0.5 \%$ bupivacaine: influence of the vertebral interspace used for injection. Br J Anaesth 1989; 62: 358-361

10. Lowson SM, Brown J, Wilkins CJ. Influence of the lumbar interspace chosen for injection on the spread of hyperbaric 0.5\% bupivacaine. Br J Anaesth 1991; 66: 465-468

11. Reynolds F. Logic in the safe practice of spinal anaesthesia. Anaesthesia 2000; 55: 1045-1046. DOI: $10.1046 / \mathrm{j} .1365-$ 2044.2000.01830.x

12. Srinivasan K, Deighan M, Crowley L, McKeating K. Spinal anaesthesia for caesarean section: an ultrasound comparison of two different landmark techniques. Int J Obstet Anesth 2014; 23: 206-212. DOI: 10.1016/j.ijoa.2014.02.004

13. Bogod D. Keeping in the Reynolds zone. Int J Obstet Anesth, 2014; 23: 201-203. DOI: 10.1016/j.ijoa.2014.05.007

14. Hoffmann VL, Vercauteren MP, Vreugde JP, Hans GH, Coppejans HC, Adriaensen HA. Posterior epidural space depth: safety of the loss of resistance and hanging drop techniques. Br J Anaesth 1999; 83: 807-809 